\title{
Lateral thoracic meningocele
}

\author{
A. E. BOOTH
}

From the Department of Neurosurgery, The Middlesex Hospital, London

A case is reported of a patient with a lateral thoracic meningocele who presented with right-sided root pain. Her symptoms responded to a posterior rhizotomy of the affected roots and obliteration of the sac of the meningocele.

\section{CASE REPORT}

Miss P.H. first presented to the Middlesex Hospital in 1955. She was then 29 years old. Her complaint was of persistent backache and occasional nausea. On examination, she showed the multiple cutaneous nodules and café-au-lait patches of von Recklinghausen's neurofibromatosis, but no neurological or other abnormality could be demonstrated. There was no family history of neurofibromatosis. No cause for her pain was found and she was discharged requiring mild analgesics.

She was next seen in 1959 with a further severe attack of her pain, intermittently present since her last admission. On this occasion she claimed that the pain was beneath the right costal margin and radiated to the tip of the right scapula. The pain was worse at night while she was in bed and often awoke her in the morning.

She again showed no abnormality on clinical examination. Spinal radiographs were reported as showing narrowing of the pedicles of D 8, 9, and 10 on the right side and a concavity of the posterior margin of the body of $\mathrm{D} 10$. There was no wedging of the vertebral bodies. The possibility of an intraspinal dumb-bell neurofibroma was raised and myelography was performed. The examination was not entirely satisfactory for the column of myodil fragmented: it was reported that there was much dilatation of the theca in the D 7-10 region but that no evidence of a neurofibroma was seen. The patient was not screened in the lateral decubitus position. Review of these films shows that a meningocele was in fact suggested, although the significance of the appearance was not appreciated. Other investigations included a barium meal, which displayed a small hiatus hernia, while a cholecystogram and an intravenous pyelogram were normal. No surgical treatment was felt to be indicated for the hiatus hernia and she was discharged taking alkalis.

In April 1968, she was again admitted to hospital. There had been recurrent attacks of pain since her previous admission and in the last 18 months she had seldom had relief. The pain was now largely in the D 910 root distribution on the right side. As before, it was at its worst at night and often awoke her in the morning. It was made worse by coughing and by leaning against the back of a chair. Again, neurological examination was substantially normal, although she was noted to have absent abdominal reflexes and some tenderness to percussion over the spines of D 9-10. A chest radiograph was normal, while straight spinal radiographs now showed some increase in the erosions of the pedicles of D 9-10. The concavity of the posterior margin of $D 10$ had increased and similar changes were now present in D 11 (Fig. 1).

The diagnosis of lateral thoracic meningocele was suggested on this appearance and was confirmed by myodil myelography. The meningocele was shown best when filled, by placing the patient in the lateral decubitus position and a postero-anterior picture taken with a horizontal ray (Fig. 2). It was shown to extend from D 8-12 on the right side, the major diverticulum being at D 10-11.

It was felt that relief of her root pain would best be achieved by sectioning the relevant posterior roots and by occluding the mouths of the dural diverticula. At operation on 11 April 1968 a wide laminectomy of D 10-12 was performed. The dural diverticula were found to consist of the dilated and much thinned root sheaths of D 11 and 12, the nerve roots lying free and under no tension within them. A posterior rhizotomy of D 11 and D 12 on the right side was performed and in addition the anterior root of D 11 was sectioned so that the larger sac could more easily be obliterated. An attempt to plicate the dilated root sheaths from their extra-dural space provoked very brisk bleeding from the extra-dural venous plexus so a fascia lata patch was cut and sutured across the mouths of the diverticula from their intradural aspect. Post-operatively the patient claimed that she was free of root pain and when last seen as an out-patient, three months after operation, confirmed that her root pain had been abolished, although she still complained of a mild midline low dorsal ache. She said that she was delighted with the result.

\section{DISCUSSION}

Lateral thoracic meningocele is a well-recognized but uncommon condition which shows a high correlation with von Recklinghausen's neurofibromatosis. Since the first description in the literature (Pohl, 1933), some 54 cases have been recorded and of these $66.7 \%$ are noted as having shown the cutaneous manifestations of the disease.

The condition is usually asymptomatic and most commonly presents as a mass in the posterior mediastinum seen on routine radiographs of the chest. Occasionally, however, both pain and 


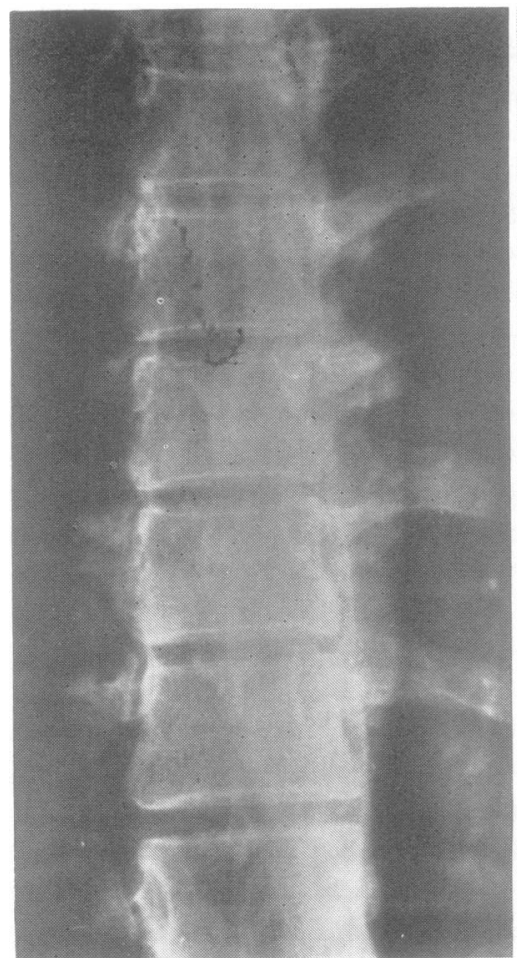

(a)

(b)

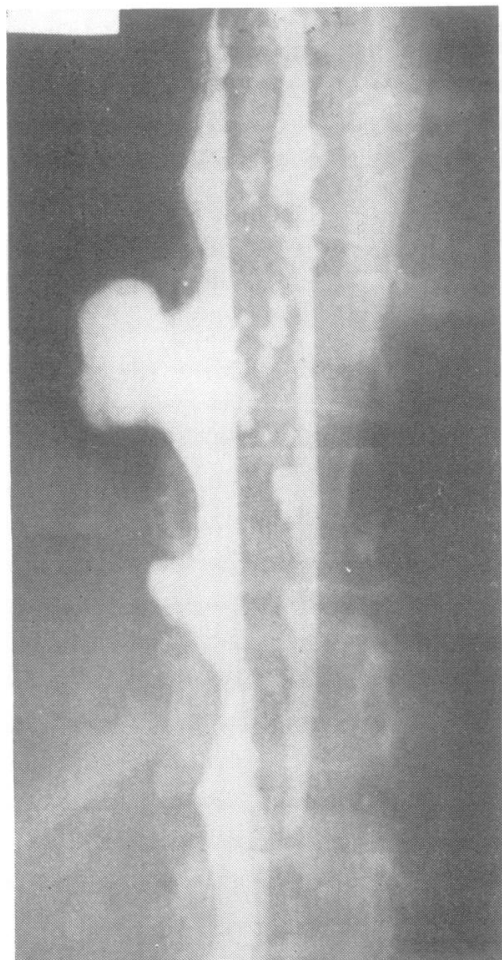

(a)
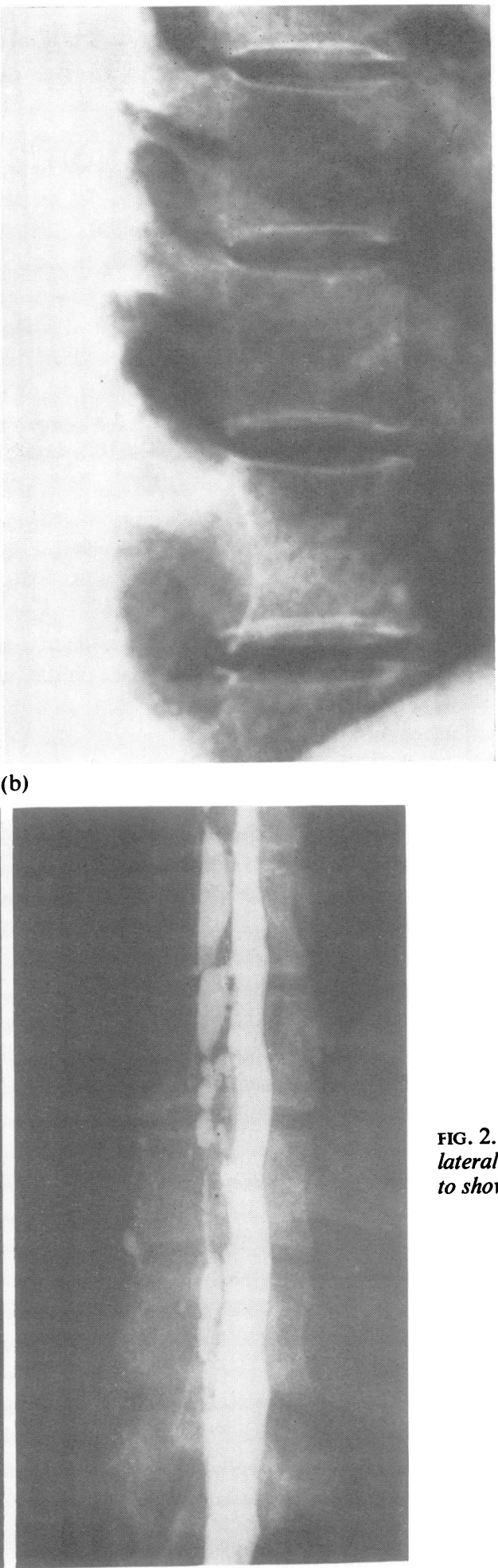

(b)
FIG. 1. Anteroposterior (a) and lateral (b) Radiography of lower dorsal spine.

FIG. 2. Right (a) and left lateral (b) decubitus myelograms to show meningocele. 
dyspnoea may be present. Dyspnoea has been reported only in patients in whom the meningocele was very large and was presumably caused by the bulk of the meningocele preventing full expansion of the lung.

The occasional reports of pain are of greater interest. It may be of two distinct types: a generalized ache in the back and, as in the present case, pain in a root distribution. Back pain of the diffuse type may reasonably be thought to be due to disturbance of the intervertebral joints secondary to the spinal deformity, for scoliosis and wedging of vertebrae are often seen. Pain may thus arise from stretched joint capsules. Thus, LaVeille and Campbell (1958) report a case in which such diffuse backache in the presence of a retrocardiac meningocele was cured by fusion of the affected vertebrae. Root pain is perhaps more difficult to explain. It is commonly held that pain in a root distribution can only be evoked by stretching and distortion of that root. O'Connell (1943) has pointed out that the intrathecal portion of a root is too easily mobile to permit such stretching, whilst the relative fixity of the root as it passes in its dural sheath between the theca and the intervertebral foramen will ensure that even a minor displacement will result in a disproportionately great increase in root tension, thus giving rise to pain. This point provides the anatomical basis for his lucid account of the production of pain in disc protrusion. It is difficult to believe that a meningocele could produce such a displacement, for the pressure in its lumen will be the same as that within the dura. Exposure of the dorsal root ganglion to the pulsatile CSF pressure after distension of the root sheath is again unlikely to produce enough irritation to give rise to pain-indeed Tarlov (1948) claims that the sacral root cysts that he has described, where pressure on the ganglia progresses to actual destruction, do not give rise to pain unless by the distortion of adjacent roots. Further, if exposure of the ganglion were itself the cause of the pain, then this should be a more common complaint in lateral thoracic meningocele where exposure of the ganglion must quite commonly occur.

It is possible that in some patients the root might be dragged laterally by the expanding sac of the meningocele. Byron, Alling, and Samson (1949) have reported a patient with a meningocele at C 7-D 1 and a history suggestive of root pain, who died at operation. At necropsy, it was found that the sac emerged between the root sheaths but that the dorsal roots of C 8 and D 1 were displaced laterally. However, the case reported by Cross, Reavis, and Saunders (1949) in which root pain was also a feature, showed at radiography rotation and subluxation of D 11 on D 12. Pain was relieved when the patient was fitted with a Boldrey brace. It would appear, therefore, that while root distortion is common to all patients complaining of root pain the mechanism of that distortion may vary. In the present case, no direct evidence of distortion of the extrathecal part of the roots was obtained and the cause of her pain must thus remain in doubt.

While neurological symptoms are unusual in this disorder, abnormal neurological signs are extremely uncommon, again reflecting the rarity of root disturbance. The only recorded patient with such signs was that of Cross et al. (1949) where hypaesthesia was recorded in that area in which he complained of pain.

Radiologically the appearances are characteristic: smooth erosion of the posterior aspects of the bodies of one or commonly more vertebrae with thinning of the pedicles on the affected side is almost always seen. The intervertebral discs are unaffected. In a few cases, erosion of the head and neck of the associated ribs has been reported, while with the larger meningoceles a smooth rounded mass may be seen behind the heart shadow and projecting into the right or left hemi-thorax. The differential diagnosis on such an appearance has been held to include a dumb-bell spinal neurofibroma. Several authorities have pointed out that such a diagnosis is unlikely: the bony changes in a lateral thoracic meningocele are seldom confined to a single vertebra or intervertebral foramen in contrast to the changes that might be expected from a solitary tumour. Kent, Blades, Valle, and Graham (1944) in a series of 105 neurogenic tumours (neurofibromas) found only four patients who showed the cutaneous manifestations of von Recklinghausen's disease. On the other hand, Hagelstam (1946) found only two cases of spinal neurofibroma in a series of 96 patients with von Recklinghausen's disease. It would thus appear that, although both the spinal neurofibromas and the cutaneous neurofibromas are manifestations of the same disease, they are seldom seen in the same patient.

The radiographic diagnosis may be confirmed by either air or myodil myelography screened in the lateral decubitus position, as illustrated in the present case. If only the prone position is used, it is unlikely that adequate filling of the sac will occur and the diagnosis may be missed.

Von Recklinghausen's disease, with its many stigmata affecting the central and peripheral nervous system, skin, and bones, may be thought to be a dysplasia both of neural crest ectoderm, whence may arise neurofibromata, glioma, and melanoma, and of mesenchyme, giving abnormalities of bone, dura, etc. Aegerter (1950) suggests that the mesenchymal disturbance is secondary to that of the tissues 
arising from the neural crest and reflects an altered nervous control probably of the blood supply of these developing tissues. He quotes Holt and Wright (1948) in support. Moore (1941) advances a similar theory.

In lateral thoracic meningocele, it is suggested that areas of dura are dysplastic and are unable to withstand the pulsatile pressures developed by the CSF in response to respiration, heart beat, etc. Herniation through intervertebral foramina and erosion of both bodies and pedicles is thus produced. Sengpiel, Ruzicka, and Lodmell (1948) comment that, in the act of coughing, the peak pressure in the CSF is delayed beyond that within the thorax, thus suggesting a further distensive mechanism. It is likely that the bony changes are secondary to the transmitted pulsation through the weakened dura rather than to a primary defect of bone. The bony changes seen have thus no part in the genesis of a meningocele. Hence, in the patient reported by LaVeille and Campbell (1958) where there were gross bony abnormalities, the meningocele has not recurred after its excision. Further, the many patients who show the bony abnormalities associated with von Recklinghausen's disease seldom show any evidence of meningocele, while in those patients known to have a lateral thoracic meningocele, the bony changes may often be shown to be progressive, as in the present case.

It is of interest that scalloping of the vertebral bodies can occur in the absence of a lateral meningocele. Thus, Howieson, Norrell, and Wilson (1968) report three cases of expansion of the subarachnoid space with marked scalloping of the posterior borders of the bodies of the vertebrae in patients who also had neurofibromatosis-indeed, one of these patients also had a small lateral thoracic meningocele. Heard and Payne (1962) report six cases of scalloping of thoracic vertebral bodies with myelographic evidence of dilatation of the dura but without any sign of lateral thoracic meningocele. It is clear that at least some of these patients showed progression of the bony changes and that a similar mechanism to that proposed for the production of lateral thoracic meningocele might well be the cause.

Views on the treatment of this condition are divided. There is general agreement that symptomatic treatment should be given, but the management of the asymptomatic patient is less uniform. Thus, Ciaglia (1952) feels that surgery is indicated in nearly all cases, pointing out that the condition is often progressive and citing the case reported by Portigliatti-Barbos (1953) where death followed the rupture of a meningocele during a fit of coughing. Others feel that conservative treatment is to be preferred, in that many of these lesions progress very slowly and may remain unchanged in size for many $\stackrel{\mathbb{C}}{D}$ years. The present author feels that in the absence of symptoms the patient should be kept under observation-the appearance of symptoms or the rapid 0 enlargement of the meningocele being taken as the indication for operation.

A transthoracic approach will provide the best access for the obliteration of the sac of a large? meningocele, causing lung compression, and will $\Rightarrow$ allow excision of the bulk of the sac. Particular care is $\stackrel{?}{+}$ necessary to prevent the formation of a CSF fistula into the thoracic cavity; imbrication of the neck of $\frac{}{\overline{0}}$. the sac and reinforcement of the suture line with $\frac{\bar{\rho}}{\sigma}$ pleura or fascia lata should give adequate protec- $\stackrel{\mathbb{Q}}{\complement}$ tion. An intradural approach to apply a patch to the neck of the sac gives the advantage that the nerve $\overrightarrow{0}$ roots may be inspected but this approach implies that the body of the sac must be left in situ. It is $\vec{\omega}$ therefore possible that a minor leak of CSF through the suture line might permit the sac to refill and, should it be large, again compress the lung.

For the relief of root pain, rhizotomy would ${ }_{N}^{\omega}$ appear to be the most satisfactory form of treatmen unless the pain is clearly secondary to the bon $\vec{\exists}$ changes. Here the approach will, of course, betrans dural. Should the meningocele be large, a furthef transthoracic approach may be necessary for its $\vec{D}$ ablation; otherwise the neck may be occluded as if the present case.

Finally, midline back pain in the presence of $\vec{\varphi}$ marked deformity may occasionally merit spinat fusion without any approach to the meningocele. There is little place for the definitive treatment of such pain with a brace.

\section{SUMMARY}

A case is described of a patient with a lateral thoracic meningocele who presented with root pain. The aetiology, symptomatology, and treatment of the condition is discussed.

My thanks are due to Dr. J. D. N. Nabarro, under whose care the patient was originally admitted, and to $\mathrm{Mr}$. J. Andrew, who performed the operation, for allowing me to record the case. I gratefully acknowledge Mr. Andrew's help and encouragement in its presentation.

\section{REFERENCES}

Aegerter, E. E. (1950). The possible relationship of neurofibromatosis congenital pseudarthrosis and fibrous dysplasia. J. Bone Jt Surg., 32A, 618-626.

Byron, F. X., Alling, E. E., and Samson, P. C. (1949). Intrathoracic meningocele. $J$. thoracic Surg., 18, 294-303.

Ciaglia, P. (1952). Intrathoracic meningocele: excision with three and one-half years follow-up. Ibid., 23, 283-292.

Cross, G. O., Reavis, J. R., and Saunders, W. W. (1949). Latera intrathoracic meningocele. J. Neurosurg., 6, 423-432. 
Hagelstam, L. (1946). On the deformities of the spine in multiple neurofibromatosis (von Recklinghausen). Acta chir. scand., 93, 169-193.

Heard, G., and Payne, E. E. (1962). Scolloping of the vertebral bodies in von Recklinghausen's disease of the nervous system (neurofibromatosis). J. Neurol. Neurosurg. Psychiat., 25, 345-351.

Holt, J. F., and Wright, E. M. (1948). The radiological features of neurofibromatosis. Radiology, 51, 647-664.

Howieson, J., Norrell, H. A., and Wilson, C. B. (1968). Expansion of the subarachnoid space in the lumbosacral region. Ibid., 90, 488-492.

Kent, E. M., Blades, B., Valle, A. R., and Graham, E. A. (1944). Intrathoracic neurogenic tumors. J. thoracic. Surg., 13, 116-161.
LaVeille, C. J., and Campbell, D. A. (1958). Neurofibromatosis and intrathoracic meningocele. Radiology, 30, 62-66.

Moore, B. H. (1941). Some orthopaedic relationships of neurofibromatosis. J. Bone Jt Surg., 23, 109-140.

O'Connell, J. E. A. (1943). Sciatica and the mechanism of the production of the clinical syndrome in protrusions of the lumbar intervertebral discs. Brit. J. Surg, 30, 315-327.

Pohl, R. (1933). Meningokele im Brustraum unter dem Bilde eines intrathorakalen Rundschattens. Röntgenpraxis, 5, 747-749.

Portigliatti-Barbos, M. (1953). Neurofibromatosi e meningocole toracico. Friuli med., 8, 477-484.

Sengpiel, G. W., Ruzicka, F. F., and Lodmell, E. A. (1948). Lateral intrathoracic meningocele Radiology, 50, 515-520.

Tarlov, I. M. (1948). Cysts (perineurial) of the sacral roots. J. Amer. med. Ass., 138, 740-744. 